\title{
Drug resistance in malaria, tuberculosis, and HIV in South East Asia: biology, programme, and policy considerations
}

\author{
Malaria, tuberculosis, and HIV present unique challenges in the control of antimicrobial resistance, \\ and require targeted policies, say Samiran Panda and colleagues
}

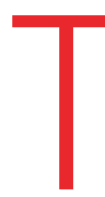

he World Health Organization South East Asia region, home to a third of the world's population, has half the global incident cases of tuberculosis (TB), and a tenth of the estimated burden of malaria and HIV. ${ }^{1}$ The risk of disease transmission from travel and migration of people from and within the region highlight the importance of tackling this large disease burden. Failure to control or eliminate these diseases could negatively affect health and development worldwide. The increasing resistance to the drugs used to treat malaria, TB, and $\mathrm{HIV}^{2}$ (fig 1), and its ability to move across national borders, are challenges to controlling these diseases. Furthermore, drug resistance in malaria, TB, and HIV, and the effect of individual, sociocultural, environmental, and political factors differ between countries, which make containment even harder.

\section{Methods}

We conducted an integrative review of the current status of malaria, TB, and HIV in the WHO South East Asia region with a focus on drug resistance and the commonalties and variations between coun-

\section{KEY MESSAGES}

- The WHO South East Asia region contributes about a half of the global incident cases of tuberculosis and a tenth of the estimated burden of malaria and

- Poor regulatory performance on antibiotic production and prescription and inappropriate use of antibiotics have compounded drug resistance in malaria, tuberculosis, and HIV in South East Asia

- The success of the Maldives and Sri Lanka in eliminating malaria offers important public health lessons for other South East Asian countries

- Key elements to infection control are national political commitment; combined prevention and treatment approaches; active engagement of the community; quality assured drugs; and strengthening of community based surveillance systems

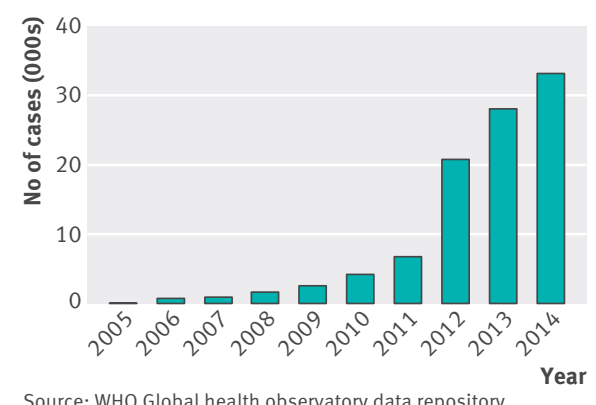

Source: WHO Global health observatory data repository

Fig 1 | Confirmed cases of rifampicin resistant or multidrug resistant tuberculosis in the WHO South East Asia region, 2005-14

tries. Our aim was to provide public health insight to facilitate policy discussion and inform programme planning at regional and national levels. We searched global and regional WHO reports, articles, and reviews published in peer reviewed journals, monographs, and unpublished grey literature. Our search focused on the biology of, and programmes and policies on, malaria, TB, and HIV. We identified the existing problems in the region and opportunities for solutions, including examples of good practices for disease prevention and control.

\section{Mechanisms of resistance}

Drug resistance (box 1) in malaria, TB, and HIV occurs either through the emergence of new resistant microorganisms or as a response to treatment (selection) pressure. Examples of response to treatment include the organism pumping a drug out of its active site ${ }^{3}$ (as happens with chloroquine in drug resistant Plasmodium falciparum malaria) and altering the site where a drug binds ${ }^{4}$ (as seen with the streptomycin binding site of Mycobacterium tuberculosis). These adaptations add to the intrinsic properties of some organisms that confer survival benefit-for example, the $M$ tuberculosis cell wall contains high levels of mycolic acid, which reduces its permeability to antibiotics and other chemotherapeutic agents. ${ }^{5}$ Antiretroviral drugs target HIV replication in the cells through three virally encoded enzymes, reverse transcriptase, protease, and integrase. With the exception of the chemokine receptor antagonists that bind to a host protein involved in viral entry, antiretroviral drugs target virally encoded structures. ${ }^{6}$ Mutations in the viral genome at the time of HIV replication confer resistance by interfering with drug binding.

An additional challenge is that re-infection and resistance can occur months or years after the first infection. In malaria, re-infection is common as previous infection does not provide complete immunity and relapse after years of treatment is possible with $P$ vivax,

\section{Box 1: Definition of drug resistance}

- Drug resistant malaria-the ability of a malaria parasite strain to survive or multiply despite the absorption of a medicine given in doses equal to, or higher than, those usually recommended

- Treatment failure-the inability to clear malarial parasitaemia or prevent recrudescence after administration of an antimalarial medicine, regardless of whether clinical symptoms are resolved. Treatment failure is not, however, always due to drug resistance and many factors can contribute, mainly by reducing drug concentrations. These factors include incorrect dosage, poor patient compliance with either dose or duration of treatment, poor drug quality, and drug interactions. Even after supervised administration of a full regimen of an antimalarial medicine, individual variations in pharmacokinetics might also lead to treatment failure because of poor absorption, rapid elimination (diarrhoea or vomiting), or poor biotransformation of prodrugs

- Multidrug resistant tuberculosis-tuberculosis that does not respond to at least isoniazid, and rifampicin, the two strongest antituberculosis drugs

- HIV drug resistance-the ability of HIV to replicate in the presence of drugs that usually suppress its replication

Source: World Health Organization 
and $P$ ovale stemming from dormant liver forms. ${ }^{7} M$ tuberculosis has a long generation time and can be dormant with low metabolic activity which makes it a difficult target for drugs. ${ }^{8}$ In addition, $M$ tuberculosis can be located in pulmonary cavities or caseous material, which are difficult for antibiotics to reach, or where low $\mathrm{pH}$ inhibits the activity of most antibiotics. ${ }^{9}$ Recent evidence suggests that HIV can continue to replicate and replenish its reservoir in the face of antiretroviral therapy. ${ }^{10}$

Social and structural factors also play a role in drug resistance, which is seen in the evolution of drug resistance in malaria in South East Asia. These include the interrupted supply of quinine during Japanese occupation in the second world war of regions where cinchona was grown, and the recent appearance of resistance to artemisinin in several areas in the region following reversal of the policy on its restricted use, and the subsequent widespread administration as the first line treatment for falciparum malaria. ${ }^{11}$ Administration of chloroquine in salt to migrant workers from surrounding countries in the mining industry in Pailin Province, Cambodia, (where mining shafts allowed the collection of water from seepage, and rain, and provided a breeding site for mosquitoes) contributed to increased drug resistance because the concentration of chloroquine in blood was not curative. The need to tackle the socioeconomic determinants of health and reach out to where the vulnerability and risk are greatest, with the active participation of the local community and committed resources for universal health coverage, have been recognised as essential for the effective containment of malaria, TB, and HIV. ${ }^{12}$

\section{Difficulties with drug treatment}

The development of drugs against malaria has been very slow and the progress made against drug resistant TB has been limited. In contrast, the development of drugs against HIV has been faster and more than 25 drugs of 6 classes are available. ${ }^{7}$ Although serious adverse effects are rare, malaria drugs are more toxic than antibacterial drugs, which can affect patients' adherence to treatment. Similarly, the first line combination drugs, which are highly effective against drug sensitive pulmonary TB, have important limitations, including: compromised efficacy in people receiving treatment outside the public health system where standard guidelines are not necessarily adhered to; toxicity of the medicines, which can affect adherence; and drug interactions such as with antiretroviral drugs. ${ }^{13}$ Reports of fake drugs in the region and self medication further compound these problems ${ }^{14}{ }^{15}$ and draw attention to the need to improve the performance of existing regulatory mechanisms.

\section{Tracking resistance}

Three types of tools are used to monitor treatment efficacy and the development of resistance to malaria, TB, and HIV drugs: clinical response after administration of quality assured drugs at recommended doses for sufficient duration; laboratory tests indicating clearance or suppression of the organisms; and high throughput molecular techniques that identify genetic mutations known to confer resistance. Only through the standardised use of these tools will it be possible to compare and interpret results over time within or between countries in the region. Currently countries in South East Asia use such tools in isolation and no best practice example of the judicious combination of these tools has yet emerged. Sentinel surveillance for drug resistance monitoring over time and geographical space to allow trend analysis is also lacking for malaria, TB, and HIV in most of the countries in South East Asia. A recent example of a molecular investigation informing a public health decision is the discovery of a new marker of artemisinin resistant falciparum malaria; a mutation at the propeller domain of the Kelch protein K13 has been associated with increased parasite survival. This has led to a new working definition of artemisinin resistance. ${ }^{16}$ On the other hand, monitoring early warning indicators of HIV drug resistance provides a record of clinic and programme performance-for example, patient adherence to anti-HIV drugs, drug supply continuity, and retention of patients on treatment-which helps give context to HIV drug resistance data obtained from national surveillance laboratories. However, a balance is needed between improvement of a comprehensive tracking system for antimicrobial resistance and drug resistance surveillance in vertical disease programmes.

\section{Public health achievements and challenges Malaria}

The confirmed cases of malaria in the WHO South East Asia region decreased from 2.8 million to 1.5 million between 2000 and 2015. Three countries accounted for over $95 \%$ of the cases in 2015-India (89\%), Indonesia (9\%), and Myanmar (2\%). ${ }^{17}$ However, drug resistance is a concern, and treatment failure with artemisinin based combination therapies has been recorded in the Greater Mekong subregion.

\section{Tuberculosis}

Treatment success rates for $\mathrm{TB}$ in the region have been more than $88 \%$ since $2009 .^{18}$
However, the region has 35\% of the global burden of multidrug resistant tuberculosis (MDR-TB). Therefore, although MDRTB occurs in fewer than 3\% of new cases, and $18 \%$ of retreatment cases, the high $\mathrm{TB}$ incidence makes these lower percentages translate into large numbers. An estimated one third of people with TB goes undetected or are treated outside the national programmes. They have poor outcomes, contribute to disease transmission, and are at greater risk of death. ${ }^{19}$ The reported prevalence of MDR-TB in 2005 was seven times higher in Burmese migrants on the Thai side of the Thai-Myanmar border than the national average for Thailand, ${ }^{20}$ highlighting the vulnerability to MDR-TB in the region from migration.

\section{HIV disease}

The emergence of HIV drug resistance, with its negative implication for individuals, society, and ongoing prevention efforts is a concern when scaling up antiretroviral therapy. However, the current evidence shows that HIV drug resistance in South East Asian countries has not yet reached a high level. A recent systematic review of studies in the region published during 2000-11 found that most studies reported low levels of HIV drug resistance. ${ }^{21}$

\section{Good practices and opportunities for cross country learning \\ Malaria}

Two countries in the region, the Maldives and Sri Lanka, are free of malaria; the Maldives since $1984^{22}$ and Sri Lanka since $2012 .{ }^{23}$ Their experiences and ability to remain malaria-free offer several public health lessons. These include (1) elimination of the parasites rather than the vector mosquitoes through adaptation of appropriate treatment guidelines, (2) risk reduction through the careful use of insecticides, (3) larvicidal measures, including insecticide rotation to prevent the development of resistance in mosquitoes, (4) ongoing training of healthcare workers, (5) active community engagement, (6) prompt identification of and interventions for imported cases, and (7) ensuring concerted vigilance, of politicians, public health doctors, general clinicians, tribal chiefs, community leaders, and community members. Population based surveillance at the household level, and surveillance in military camps and difficult geographical terrain, were crucial for all these interventions to work together.

\section{Tuberculosis}

Examples of good practice in the region for TB include: embedding operational research in the national disease control programme, and using research findings 
to inform evidence based policy making; ${ }^{24}$ the introduction of a rapid diagnostic test kit in Indonesia which led to a considerable increase in the detection and treatment of resistant TB cases; and the establishment of a web based case notification system in India. The Indian system of notification, under the revised national TB control programme, also included private health providers (http://nikshay.gov.in/AboutNikshay. $\mathrm{htm})$. Ministers of health of all the $11 \mathrm{mem}$ ber countries of the WHO South East Asia region also called upon leaders, policy makers, partners, civil society, and the public in the region and around the world actively to support their call for action to end TB (http://www.searo.who.int/tb/call_for_ action_signed_v_1.pdf?ua=1). The two key commitments of the ministers under this call were to lead the national TB response through an empowered body reporting to the highest level of government, and to increase government and partner budget allocations to enable national TB plans to be fully funded.

\section{HIV disease}

The HIV drug resistance laboratory at the National AIDS Research Institute in India is a WHO accredited laboratory for HIV drug resistance genotyping. As part of its technical assistance, WHO has established links between this research institute and government health authorities in Sri Lanka, Myanmar, and Nepal. Through this link, Sri Lanka has been conducting a study since 2015 to help clinical decision making for patient management, and Myanmar completed a survey during 2016-17 to identify primary HIV drug resistance. A similar survey on acquired and primary drug resistance is ongoing in Nepal. In addition, the National Research Institute in India has undertaken important research within countries, for example on HIV drug resistance among treatment naive children. ${ }^{25}$

\section{Way forward}

The three epidemiological factors (agent, host, and environment) associated with malaria, TB, and HIV are constantly changing. Dealing with these changes in order to contain these diseases requires innovative approaches, one of which is adaptation of combination therapy. This therapeutic strategy is based on the understanding that the probability of an organism developing resistance against multiple drugs that have different mechanisms of action is the product of the probability of a drug resistance mutation developing in the organism against each component, and such co-occurrence is rare. Shorter duration of treatment for MDR-TB ( 9 months compared with the earlier regimen of 2 years), based on the results of field trials, is being considered as another pragmatic approach. On the other hand, the lack of combined essential services for vulnerable groups, for example needle and syringe exchange, oral substitution therapy, and antiretroviral drugs for people who inject drugs, is an important service gap and has been used to advocate for policy and programme change in the region. ${ }^{26}$

In conclusion, vector control, or behavioural risk reduction measures alone are not enough for disease prevention, and combination treatment should be used at the same time. However, other determinants of health, such as poverty, underdevelopment, and stigma, if not also tackled, will continue to leave some population groups vulnerable to disease. We think therefore that the following five elements are important for the effective control or elimination of malaria, TB, and HIV: commitment and ownership at the country level; combination approach in prevention and treatment; community engagement, including participation of vulnerable groups (people who inject drugs, mobile and migrant populations, residents of labour settlements); care of patients with quality assured, effective medicines, and necessary regulatory measures; and decentralised surveillance systems to detect community spread of infections early.

Contributors and sources: SP, SS, KAH, EMC, RNP, TYA, PS, ABT, and SKS conceived the idea. SP, ANS, SJL, and RS wrote the manuscript. TYA, ABT, GBN and SKS edited the manuscript. All authors have read and approved the manuscript.

Competing interest: We have read and understood BMJ policy on declaration of interests and have no relevant interests to declare.

Provenance and peer review: Commissioned, externally peer reviewed.

This article is one of a series commissioned by The $B M$ J based on an idea from WHO SEARO. The BMJ retained full editorial control over external peer review, editing, and publication. Open access fees are funded by the WHO SEARO.

Samiran Panda, senior deputy director

Soumya Swaminathan, director general ${ }^{2}$ Khurshid A Hyder, regional adviser tuberculosis ${ }^{3}$ Eva-Maria Christophel, regional adviser malaria ${ }^{3}$ Razia $\mathrm{N}$ Pendse, WHO representative ${ }^{4}$

Achuthan N Sreenivas, national professional officer Setiwan J Laksono, national professional officer ${ }^{6}$ Rahul Srivastava, junior public health professional ${ }^{3}$ Gopinath B Nair, acting regional adviser research policy and cooperation ${ }^{3}$

Tjandra Y Aditama, special adviser to the regional director $^{3}$

Pratap Singhasivanon, dean

Arun B Thapa, director programme management ${ }^{3}$ Swarup K Sarkar, director communicable diseases ${ }^{3}$

${ }^{1}$ ICMR-National Institute of Cholera and Enteric Diseases, Kolkata, India

${ }^{2}$ Indian Council for Medical Research, Government of India, New Delhi, India

${ }^{3}$ World Health Organization, Regional Office for SouthEast Asia, New Delhi, India
${ }^{4}$ WHO Representative, World Health Organization Country Office, Colombo, Sri Lanka

${ }^{5}$ World Health Organization Country Office, New Delhi,

${ }^{6}$ World Health Organization Country Office, Jakarta, Indonesia

${ }^{7}$ Faculty of Tropical Medicine, Mahidol University, Bangkok, Thailand

Correspondence to:SKSarkar

sarkarswa@who.int

World Health Organization. Global health observatory data repository. Population data by WHO region. 2015. http://apps.who.int/gho/data/view.main. POP2020.

Sharma A, Hill A, Kurbatova E, et al. Global Preserving Effective TB Treatment Study Investigators. Estimating the future burden of multidrug-resistant and extensively drug-resistant tuberculosis in India, the Philippines, Russia, and South Africa: a mathematical modelling study. Lancet Infect Dis 2017:17:707-15. doi:10.1016/S1473-3099(17)30247-5

3 Koenderink JB, Kavishe RA, Rijpma SR, Russel FG The ABCs of multidrug resistance in malaria. Trends Parasitol 2010;26:440-6. doi:10.1016/j. pt.2010.05.002

4 Almeida Da Silva PE, Palomino JC. Molecular basis and mechanisms of drug resistance in Mycobacterium tuberculosis: classical and new drugs. J Antimicrob Chemother 2011;66:1417-30. doi:10.1093/jac/dkr173

Jarlier V Nikaido H. Mycobacterial cell wall: structure and role in natural resistance to antibiotics. FEMS Microbiol Lett 1994;123:11-8. doi:10.1111/j.1574-6968.1994.tb07194.x

6 Johnson VA, Brun-Vézinet F, Clotet B, et al. Update of the drug resistance mutations in HIV-1: December 2010. Top HIV Med 2010;18:156-63.

Goldberg DE, Siliciano RF, Jacobs WRJr. Outwitting evolution: fighting drug-resistant TB, malaria, and HIV. Cell 2012;148:1271-83. doi:10.1016/j. cell.2012.02.021

8 Wayne LG. Dormancy of Mycobacterium tuberculosis and latency of disease. Eur J Clin Microbiol Infect Dis 1994:13:908-14. doi:10.1007/BF02111491

9 Elliott AM, Berning SE, Iseman MD, Peloquin CA. Failure of drug penetration and acquisition of drug resistance in chronic tuberculous empyema. Tuber Lung Dis 1995;76:463-7. doi:10.1016/0962 8479(95)90016-0

10 Lorenzo-Redondo R, Fryer HR, Bedford T, et al. Persistent HIV-1 replication maintains the tissue reservoir during therapy. Nature 2016;530:51-6. doi:10.1038/nature16933

11 Packard RM. The origins of antimalarial-drug resistance. N Engl J Med 2014;371:397-9. doi:10.1056/NEJMp1403340

12 World Health Organization, Regional Office for South-East Asia. End in sight: accelerating the end of HIV, TB and malaria, and neglected tropical diseases in the South-East Asia Region. 2016. http://apps.who.int/iris/bitstre am/10665/249586/1/9789290225270-Eng. pdf?ua $=1$

13 Yee D, Valiquette C, Pelletier M, Parisien I, Rocher I, Menzies D. Incidence of serious side effects from first-line antituberculosis drugs among patients treated for active tuberculosis. Am / Respir Crit Care Med 2003;167:1472-7. doi:10.1164/ rccm.200206-6260C

14 Dondorp AM, Newton PN, Mayxay M, et al. Fake antimalarials in Southeast Asia are a major impediment to malaria control: multinational cross-sectional survey on the prevalence of fake antimalarials. Trop Med Int Health 2004;9:1241-6. doi:10.1111/j.1365-3156.2004.01342x

15 Bhatia R, Narain JP. The growing challenge of antimicrobial resistance in the South-East Asia Region-are we losing the battle? Indian / Med Res 2010;132:482-6

16 World Health Organization. Artemisinin and artemisinin-based combination therapy resistance. Status report. WHO, 2017. http://apps.who.int/ 
iris/bitstream/10665/255213/1/WHO-HTM-GMP2017.9-eng.pdf

17 World Health Organization. Global malaria report 2015. 2015. http://apps.who.int/iris/ bitstream/10665/200018/1/9789241565158_ eng.pdf?ua $=1$

18 World Health Organization Regional Office for SouthEast Asia. Tuberculosis control in the South-East Asia Region: annual report 2016. 2016. http://apps.who. int/iris/handle/10665/205286

19 Nair N, Wares F, Sahu S, et al. Tuberculosis in the WHO South-East Asia Region. Bull World Health Organ 2010;88:164. doi:10.2471/ BLT.09.073874

20 Beyrer C, Suwanvanichkij V, Mullany LC. Responding to AIDS, tuberculosis, malaria, and emerging infectious diseases in Burma: dilemmas of policy and practice. PLoS Med 2006;3:e393. doi:10.1371/ journal.pmed.0030393

21 Trotter AB, Hong SY, Srikantiah P, Abeyewickreme I, Bertagnolio S, Jordan MR. Systematic review of HIV drug resistance in Southeast Asia. AIDS Rev 2013:15:162-70.

22 World Health Organization Regional Office for South-East Asia. Malaria-free Maldives. 2016. http://apps.who.int/iris/bitstre am/10665/250397/1/9789290225140-eng. pdf?ua $=1$

23 World Health Organization Regional Office for South-East Asia. Malaria-free Sri Lanka. 2016. http://apps.who.int/ iris/bitstream/10665/251824/1/9789290225423eng.pdf?ua $=1$

24 Mahendradhata Y, Probandari A, Widjanarko B, et al. Embedding operational research into national disease control programme: lessons from 10 years of experience in Indonesia. Glob Health Action 2014;7:25412. doi:10.3402/gha. v7.25412

25 Kumar M, Sen S, Gupta RK, Chaturbhuj D, Tripathy SP, Paranjape RS. Prevalence of drug resistance in human immunodeficiency virus type 1-infected treatment-naive children in Pune, India. AIDS Res Hum Retroviruses 2014;30:294-8. doi:10.1089/aid.2013.0222.

26 Panda S, Kumar MS. Injecting drug use in India and the need for policy and program change. Int J Drug Policy 2016;37:115-6. doi:10.1016/j. drugpo.2016.08.009.

Cite this as: BM/ 2017;358:j3545 http://dx.doi.org/10.1136/bmj.j3545

This an open access article distributed under the terms of the Creative Commons Attribution-non-commercial IGO License (https:// creativecommons.org/licenses/by-nc/3.0/igo/), which permits use, distribution, and reproduction for non-commercial purposes in any medium, provided the original work is properly cited. In any reproduction of this article there should not be any suggestion that WHO or this article endorse any specific organisation or products. The use of the WHO logo is not permitted. This notice should be preserved along with the article's original URL. 